\title{
Reciprocal Translocations in Cattle: frequency estimation
}

Lisa De Lorenzi ${ }^{1}$, Paola Morando ${ }^{2}$, Jordi Planas ${ }^{3}$, Michele Zannotti ${ }^{1}$, Luciano

Molteni $^{1}$ \& Pietro Parma ${ }^{1}$

${ }^{1}$ Department of Animal Science, Milan University, Milan, Italy

${ }^{2}$ Department of Agricultural Engineering, Milan University, Milan, Italy

${ }^{3}$ Departement of Systems Biology, Vic University, Vic, Spain

\section{Correspondence}

Pietro Parma,

Dipartimento di Scienze Animali, Sezione di Zootecnica Agraria, Via Celoria 2, 20133 Milano, Italia.

Tel. +390250316454

Fax. +390250316434

Email pietro.parma@unimi.it 


\section{Summary}

Chromosomal anomalies, like Robertsonian and reciprocal translocations represent a big problem in cattle breeding as their presence induces, in the carrier subjects, a well documented fertility reduction. In cattle reciprocal translocations (RCPs, a chromosome abnormality caused by an exchange of material between nonhomologous chromosomes) are considered rare as to date only 19 reciprocal translocations have been described. In cattle it is common knowledge that the Robertsonian translocations represent the most common cytogenetic anomalies, and this is probably due to the existence of the endemic 1;29 Robertsonian translocation. However, these considerations are based on data obtained using techniques that are unable to identify all reciprocal translocations and thus their frequency is clearly underestimated. The purpose of this work is to provide a first realistic estimate of the impact of RCPs in the cattle population studied, trying to eliminate the factors which have caused an underestimation of their frequency so far. We performed this work using a mathematical as well as a simulation approach and, as biological data, we considered the cytogenetic results obtained in the last 15 years. The results obtained show that only $16 \%$ of reciprocal translocations can be detected using simple Giemsa techniques and consequently they could be present in no less than $0,14 \%$ of cattle subjects, a frequency five times higher than that shown by de novo Robertsonian translocations. This data is useful to open a debate about the need to introduce a more efficient method to identify RCP in cattle. 


\section{Introduction}

"In cattle reciprocal translocations are rare". This is the most common opening statement in scientific papers describing reciprocal translocations (RCPs) in cattle; furthermore this statement is often followed by another: "In cattle, Robertsonian translocations (ROBs) are more frequent than RCPs". Are these two statements true? In human newborns, the frequency of these anomalies are very similar: $0.10 \%$ and $0.08 \%$ (RCPs and ROBs respectively; Van Assche et al., 2006) and these frequencies are higher in couples experiencing repeated pregnancy losses (1.07\% RCPs and $0.81 \%$ ROBs) (De Braekeleer and Dao, 1991). In cattle the incidence of these two anomalies is difficult to determine, as several parameters must be considered. During the Italian official cytogenetic screening program (22,735 animals studied over 15 years, Supplementary Table 1) 1,609 carriers of ROBs (7.1\%) and 5 RCP carriers (0.03\%) were discovered (Ducos et al., 2008 and unpublished data from the authors). Nevertheless $99.6 \%$ of ROBs is represented by endemic 1;29 (none de-novo) and only 6 subjects ( $0.03 \%)$ were discovered to be carriers of ROB translocation excluding $1 / 29$. Thus both kinds of anomalies show a very similar frequency of incidence.

A correct frequency estimation of these two kinds of anomalies (in contrast with other species such as humans and pigs) must consider the structure of cattle karyotype: all 29 autosomal pairs possess only one arm and consequently centric fusions could be more favourable (one armed chromosomes are a small part of human and pig karyotype). Therefore it is 
credible to state that in cattle $\mathrm{ROB}$ frequency is higher than $\mathrm{RCP}$, but it is certainly true that RCP frequency is undervalued.

ROBs and RCPs are responsible for significant economic losses (Dyrendahl and Gustavsson, 1979; Schmutz et al, 1996; Lonergan et al., 1994; Schmutz et al., 1991; Rodriguez et al., 2010; Makinene et al., 1997) and thus their identification in animals intended for reproduction represents an important step in the modern genetic selection programs. Usually screening programs are mainly performed using the GIEMSA standard staining, as the application of other time consuming techniques available is not suitable for a large number of analyses, consequently chromosomal exchanges between two or more chromosomes are not easy to detect in cattle, unless they produce derivative chromosomes what can be more easily observed in metaphases. Routine GIEMSA standard staining indeed allows the identification of RCP only in the presence of chromosomes that are longer or shorter than the largest and smallest chromosome. Furthermore time consuming banding techniques cannot identify RCPs involving a) small chromosomes or b) small parts of chromosomes.

The aim of this work is to find an answer to the following question: How often could reciprocal translocations occur in cattle? To answer this apparently simple question, we performed both a simulation and a mathematical approach that, together with the experimental data obtained during the cytogenetic screening performed in our lab, provide a "realistic value". 


\section{Materials and Methods}

\section{Bases of the procedure applied}

The actual frequency of RCP carriers (AF) in the cattle population studied is:

[1] $\mathrm{AF}=\frac{\mathrm{NC}}{\mathrm{NA}}=\frac{\mathrm{NI}+\mathrm{NL}}{\mathrm{NA}}$

where: $\quad N C=$ total number of individuals actually carrying a RCP

$$
\begin{aligned}
& \mathrm{NA}=\text { total number of individuals controlled } \\
& \mathrm{NI}=\text { number of carriers detected } \\
& \mathrm{NL}=\text { number of carriers not detected }
\end{aligned}
$$

Therefore in order to solve this formula, we must estimate the number of RCPs that were not observed (NL).

[2] $\mathrm{NL}=\frac{\mathrm{NI}}{\mathrm{PD}}-\mathrm{NI}$

where: $\quad \mathrm{NL}=$ number of carriers not detected

$$
\begin{aligned}
& \mathrm{NI}=\text { number of carriers detected } \\
& \mathrm{PD}=\text { probability of detection }
\end{aligned}
$$

and the unknown parameter is:

[3] $\mathrm{PD}=\frac{\mathrm{NIT}}{\mathrm{N}}$

where: $\quad P D=$ probability of detection

$\mathrm{NIT}=$ total number of detectable RCPs

$\mathrm{N}=$ total number of possible RCPs (that can theoretically occur) finally NIT for a particular chromosome combination -NITP- (among the 406 possible RCPs as we considered the 29 autosomal chromosomes only) is [4] NITP $=$ DP $\times$ NP

where: $\quad$ NITP $=$ total number of detectable RCPS for a particular chromosome combination 


$$
\begin{aligned}
\mathrm{DP}= & \text { proportion of the RCPs involving a particular chromosome } \\
& \text { combination that are detectable } \\
\mathrm{NP}= & \text { number of RCP involving a particular chromosome } \\
& \text { combination over a total of } 10,000 \text { theoretically possible } \\
& \text { RCPs. }
\end{aligned}
$$

finally the total number of detectable RCPs will be:

[5] NIT $=\sum_{i=0}^{406}$ NITPi

\section{Estimation of Proportion of detectable RCPs (DP)}

It is widely known that, by applying GIEMSA standard staining, a RCP can be identified only if it produces at least one derivative longer than BTA1 or shorter than BTA29. BTA1 is $161 \mathrm{Mb}$ long whereas, from a genomic point of view, the shortest cattle chromosome is not BTA29, as commonly indicated for cytogenetic purposes, but it is BTA25 (44 Mb long; data obtained from USCS genome web browser, Bta_4 assembly). Consequently, we can state that an RCP is identifiable only if it produces a derivative longer than $161 \mathrm{Mb}$ or shorter than $44 \mathrm{Mb}$. These two parameters are considered only to develop a simulation approach whereas more realistic parameters will be considered later on. An RCP is caused by the formation of breakpoints on two or more different chromosomes and the consequential exchange of genetic materials: but where do these breaks happen? Due to the scarcity of RCP data in cattle (Supplementary Methods 1), which does not allow an accurate evaluation of breakpoints distribution, we considered, as in humans (Cohen et al., 1996), that the breakpoints may arise randomly along the chromosomes excluding the presence of hot spots. 


\section{Simulation approach}

In order to determine the frequency of detectable RCPs by computer simulation we proceeded in three steps. First, we calculated what we will, from now on, refer to as the "probability of detection" for each chromosome pairing. Next, we calculated the frequency of each pairing among all possible options of pairings. Finally, the frequency of detectable RCPs is the sum of all pairings of the products of the frequency of detectability and the probability of translocation in each pairing. We have implemented this process in a PERL script and we have developed a web interface (http://www.systemsbiology.cat/) where the user can check the frequency of identifiability changes by changing some parameters as we describe below.

In order to estimate the frequency of detectability, we simulated the natural process of pairing chromosomes in all possible 406 combinations. Then, for each pairing we randomly chose a breaking point in each of the paired chromosomes using a random number generator in PERL, and pasted together the resulting fragments as in a natural translocation. The length of the two virtually translocated chromosomes is then measured and compared with both, the length of BTA1 and that of BTA25. If the length of one of the two resulting chromosomes is longer than BTA1 or shorter than BTA 25 then we consider that this translocation can be identified. However, the algorithm allows the identification of translocated chromosomes that have a difference in length of only one base pair compared to the reference chromosomes, which is an unrealistic approximation of the discrimination capacity of a human operator. We introduced the possibility to correct the discrimination 
capacity of an operator by conditioning the identification of translocations which exceed a certain difference in length. In order to obtain reasonable estimates of the frequency of identifiability, the program performs ten thousand iterations for each chromosome pairing.

\section{Mathematical approach}

In this section we explain, from a mathematical point of view, the statement that an RCP is identifiable only if it produces a derivative longer than $M$ or shorter than $m$ (a more detailed explanation is reported in Supplementary Methods 2). We start by fixing a couple of chromosomes $A$ and $B$, and we denote by $a$ and $b$ their length (without loss of generality we suppose $a \leq b$ ). Then we consider the couple $(x, y)$ with $0 \leq x \leq a$ and $0 \leq y \leq b$ : this corresponds to possible breakpoints $x$ on the chromosome $A$ and $y$ on the chromosome $B$. The lengths of derivative chromosomes $\operatorname{der}(A)$ and $\operatorname{der}(B)$, than appear consequent to the exchange of genetic materials in the corresponding $\mathrm{RCP}(A, B)$, are $x+(b-y)$ and $y+(a-x)$ respectively.

It is known that the couple $(x, y)$ generates a detectable RCP if and only if at least one new short or one new long chromosome appears, i.e. one of the following two conditions is satisfied:

$$
\begin{aligned}
& \text { (1) } x+(b-y) \leq m \text { or } y+(a-x) \leq m \\
& \text { (2) } x+(b-y) \geq M \text { or } y+(a-x) \geq M
\end{aligned}
$$

This means that detectable RCPs are in one to one correspondence with the points of the rectangle $R=\left\{(x, y) \in R^{2} \mid 0 \leq x \leq a, 0 \leq y \leq b\right\}$ satisfying conditions (1) and (2). Thus the probability that an RCP is detectable can be calculated 
as the area of part of the rectangle $R$ satisfying condition (1) or (2) over the area of the whole rectangle $R$ (equal to $a b$ ).

It is easy to check that condition (1) identifies two triangular regions belonging to $R$. Since the area of each region is equal to $\frac{m^{2}}{2}$, the probability that the $\operatorname{RCP}(A, B)$ is detectable because of the appearance of a short new chromosome is given by $\frac{1}{a b}\left(\frac{m^{2}}{2}+\frac{m^{2}}{2}\right)=\frac{m^{2}}{a b}$.

On the other side, analyzing condition (2), it is easy to demonstrate that for $a+b<M$ the straight lines $y=x-(M-b)$ and $y=x+(M-a)$ do not intersect the rectangle $R$ (i.e. there are no $\operatorname{RCP}(A, B)$ generating a new long chromosome).

On the other hand, if $a$ and $b$ are such that $a+b \geq M$, the points $(x, y)$ satisfying condition (2) fall into two triangles, each one with area equal to $\frac{(a+b-M)^{2}}{2}$

In this case, the probability that the $\operatorname{RCP}(A, B)$ is detectable due to the appearance of at least one new chromosome longer than $M$ is given by $\frac{(a+b-M)^{2}}{a b}$.

At this point are interested in determining the probability that the $\operatorname{RCP}(A, B)$ generates at least one new chromosome longer than $M$ or shorter than $m$. This means that we have to compute the area of the union of triangular sets identified by conditions (1) and (2). Since in general these triangles have no empty intersections, we can conclude that the probability for the $\operatorname{RCP}(A, B)$ to 
be detectable is given by the maximum between $\frac{m^{2}}{a b}$ and $\frac{(a+b-M)^{2}}{a b}$. A simple computation shows that, given two chromosomes $A$ and $B$ of length $a$ and $b$ respectively, the probability for the $\operatorname{RCP}(A, B)$ to be detectable (generating at least one new chromosome longer than $M$ or shorter than $m$ ) is given by

$$
\begin{aligned}
& \text { - } \frac{m^{2}}{a b} \quad \text { if } a+b<M+m \\
& \text { - } \frac{(a+b-M)^{2}}{a b} \quad \text { if } a+b \geq M+m .
\end{aligned}
$$

Estimation of the number of RCPs involving a particular chromosome combination over a total of 10,000 theoretically possible RCPs (NP)

Considering only the autosomal chromosomes, 406 different RCPs are theoretically possible $(1 ; 2,1 ; 3, \ldots, 27 ; 28,27 ; 29$ and $28 ; 29)$. An aspect to consider is the estimation of the frequency with which these different RCPs can occur. In humans it has been proposed that the involvement of the different chromosomes is proportional to the number of $R$ bands (Warburton et al., 1991). This rule is valid for most of the 23 human chromosomes, but not for all as some chromosomes are involved more than expected and others less than expected (Cohen et al., 1996). This is due in part to the existence of a recurrent RCP $(11 ; 21$, Fraccaro et al., 1980) and to other unknown factors. Considering that cattle RCPs are too few to estimate a distribution frequency the choice of the parameter to use to estimate the frequency of each possible $\mathrm{RCP}$ in cattle is the next challenge. We considered three different parameters: a) Chromosome length in $\mathrm{Mb}$; b) Number of $\mathrm{R}$ band and c) Summa of the 
length of $R$ band. The last value was obtained by measuring the chromosomes and $\mathrm{R}$ bands length from the cattle standard karyotype (Cribiu et al., 2001). The results obtained are reported in Supplementary Table 2. We can observe that all three parameters give quite a similar forecast of chromosome involvement in a RCP and, as we will report later, the final result is not dependent of the parameter used. Thus we decided to use parameter b) in the on process (the more a chromosome is rich in $\mathrm{R}$ bands the more it has the opportunity to be involved in a RCP).

The supposed frequency of each RCP is calculated using the following formula:

$\operatorname{Frequency}(R C P(i, j))=\frac{\lambda_{i} \lambda_{j}}{\sum_{i \neq j} \lambda_{i} \lambda_{j}}$

The results are reported in Supplementary Table 3 . The most frequently forecast RCP is RCP 1;2 (68.70 times over 10,000 hypothetical random RCPs).

\section{Results}

\section{Estimation of Proportion of detectable RCPs (DP)}

Both approaches give the same results. Supplementary Table 4 shows the percentage of theoretical identifiability of each of the possible 406 RPCs (theoric DP). The values vary from a maximum of $87.6 \%$ for $R C P 1 ; 2$ to a minimum of $19,3 \%$ for RCP $4 ; 14$. It is interesting to note that RCPs involving chromosomes of medium size are the least identifiable because they have less opportunity to produce a derivative with the characteristics that are useful for observation. However, this data is purely theoretical as no researcher is 
able to identify a derivative chromosome 1 base longer than BTA1 or 1 base shorter than BTA29 observing a GIEMSA stained metaphase. Consequently these values must be recalculated taking into account the ability to identify a derivative chromosome. We have performed several tests on artificially modified metaphases (Figure 1) and we have established that it is possible to suspect the presence of a chromosomal anomaly, and therefore expand the investigations, only if a derivative is $15 \%$ longer than BTA1 (185.2 Mb) or $40 \%$ shorter than BTA25 (26.4 Mb). We have chosen this test as each operator can verify their own ability to identify a chromosome anomalous in length (see (http://www.systems-biology.cat/) . An up to date analysis of identified RCPs would have been ineffective because thay all produced a derivative clearly greater than the BTA1 (from $+15 \%$ to $+28 \%$; Supplementary methods 1 ).

These two parameters depend a) on the skill of the operator: the more experianced an operator is the more he will be able to identify "short" derivative chromosomes; and b) on the condensation degree of the metaphases observed.

Detectability of the possible RCPs using these two parameters (realistic DP) is shown in Supplementary Table 5 . We can note how detectability of each RCP clearly decreases: only $60,1 \%$ of RCPs involving BTA1 and BTA2 could be detected and only $6,6 \%$ of RCPs $4 ; 15$ could be observed.

\section{Evaluation of lost RCPs}

The number of RCPs identifiable is therefore obtained by multiplying the frequency (over 10,000 random RCPs) of each RCP for its identifiability as reported in formula [4]. For example NIT for RCP $1 ; 2$ is 


$$
\begin{aligned}
& \operatorname{NITP}_{1 ; 2}=\mathrm{DP}_{1 ; 2} \times \mathrm{NP}_{1 ; 2} \\
& \text { NITP }_{1 ; 2}=0.601 \times 68.70=41.32
\end{aligned}
$$

The results are reported in Supplementary Table 6.

The sum of all identifiable RCPs (formula [5]) gives the total number of detectable RCPs: 1593.09 .

Consequently formula [3] is:

$$
P D=1,593.09 / 10,000=0.1593
$$

and considering that to date only five have been observed we believe that around 26.4 RCPs escaped observation (as described in formula [2].

$$
\mathrm{NL}=(\mathrm{NI} / \mathrm{PD})-\mathrm{NI}=\mathrm{NL}=(5 / 0.1593)-5=26.4
$$

This data allows us to estimate the frequency of RCP in the cattle population studied as (formula [1]):

$$
\mathrm{AF}=\frac{\mathrm{NC}}{\mathrm{NA}}=\frac{\mathrm{NI}+\mathrm{NL}}{\mathrm{NA}}=(5+26.4) / 22,735=0.0014
$$

and $0,14 \%$, is a frequency apparently five times higher than that shown by de novo ROBs.

\section{Discussion}

In this work we provide two results: a) we estimate that using the widely used Giemsa technique only $16 \%$ of RCPs can be detected; b) we esteem that in the cattle population considered, approx. 1.4 subjects over 10,000 could be RCP carriers. Of course this estimate still has to be validated and the only rigorous way to do this is to carry out large scale, and expensive, banding based cytogenetic screening. 
Chromosomal abnormalities such as ROB and RCP translocations, are responsible for important economic losses as carriers produce unbalanced gametes that have a high probability of producing non-viable embryos.

The influence of these types of chromosomal anomalies on fertility is widely acknowledged, but some differences exist between sex and between ROB and RCP.

In cattle carrying ROB $1 ; 29$ the observed value of unbalanced sperm is $2.76 \%$ in sperms and $4.06 \%$ in oocytes (Bonnet-Garnier et al. 2008). This is true for ROB 1;29 but other ROBs can follow other segregation profiles between balanced and unbalanced gametes. In humans the unbalanced sperm proportion in several ROB types ranges from 10 to $24 \%$ (Ogur et al., 2006). In addition the effect on fertility could be incremented considering that the segregation of other chromosomes, mainly sex chromosomes) is influenced by the presence of a ROB translocation (Ogur et al., 2006).

In the presence of a RCP segregation studies show that the \% of unbalanced sperm is greater than that reported for ROB.

In pig several studies show that the proportion of unbalanced sperm ranges from $47.83 \%$ to $24.33 \%$ depending on RCP type (Pinton et al., 2004). Moreover the segregation seems to be independent on time and subjects (Massip et al., 2005). Studies performed on females show that in the case of a particular RCP, 3;15(q27;q13) the proportion of unbalanced gametes is lower compared to male: 35.4 vs $47.83 \%$ (Pinton et al., 2005).

The dependence on RCP type of \% of unbalanced gametes is confirmed in human studies where the proportion of chromosomally unbalanced sperm 
produced by translocation carriers can range from $19 \%$ to more than $80 \%$, and appears to be dependent on the translocation (reviewed in Benet et al., 2005, Morel et al., 2004).

Moreover the presence of a RCP can induce failure in the formation of synaptonemal complex and an arrest of meiosis process (Gonsalves et al., 2004; Fergusson et al., 2008.

Thus it is clear that the influence on fertility shown by RCP is greater than that shown by ROB. Unfortunately there is no data about sperm or oocyte segregation of RCP carriers to date.

Using the procedure described we propose that 1.4 subjects over 1,000 are carriers of a reciprocal translocation. This value is higher than reported for de novo $\mathrm{ROB}(0.03 \%$ if $\mathrm{ROB} 1 ; 29$ is excluded). Furthermore we consider this value the lowest possible value as taking into account other parameters can only increase this value. We know that a visible recurrent cattle RCP does not exist (as RCP 11;22 in humans), and the eventual existence of an "invisible" RCP would only increase this percentage. Another factor tha has not been considered is represented by fragile sites. In humans chromosomes carrying fragile site show more inclination to be involved in RCP than expected (Cohen et al., 1996). In cattle various fragile sites were identified (Rodriguez et al., 2002) thus we must consider the eventuality that these sites are more frequently involved in RCP formation. Moreover if we presume the existence of an RCP deriving from two fragile site breakpoints, the likelihood of being visible is very low. Calculating the \% of detectability of each possible RCP only $6.84 \%$ of them produce derivative chromosomes $15 \%$ longer than BTA1 or $40 \%$ shorter than BTA25 (data not shown). 
Finally in humans $45 \%$ of $\mathrm{R}$ band are located in proximal region and consequently a larger part of breakpoints are expected to occur inside this region. However the analysis of a large number of RCPs shows that chromosome ruptures occur along the whole chromosome with the same probability. In cattle $\mathrm{R}$ bands are equally distributed over all chromosome length (data not shown) even if their area seems to be more concentrated in distal position rather then proximal and median. Simulation studies reveal that presuming random breakpoints map not along the whole chromosome length but on its distal part, the number of detectable RCPs falls. Consequently if at least one of these factors exist, it will induce an augmentation of the proposed value of $0,14 \%$ carrier subjects.

This data is useful to open a debate about the need to introduce into routine analyses of cattle a more efficient method to identify RCP, considering the higher cost of these analyses compared to conventional GIEMSA technique. A cytogenetic analysis performed with GIEMSA standard staining costs around $40-50 €$ whereas a karyotype analysis involving RBG banding is evaluated at no less than $350-450 €$ : this is principally due to the time necessary to assemble a banded cattle karyotype (personal communication from the Authors). Perhaps these more detailed analyses are not justified in all animals destined for reproduction, but only in animals that positively pass the progeny test and are close to start sperm production for artificial insemination procedure. The need to perform more detailed cytogenetic analyses is also justified with the advent of the "genomic evaluation" in cattle population. This procedure will soon lead to the dropping of progeny testing 
procedure (Goddard et al., 2010) and this will increase the spread of chromosome rearrangement in the population.

Finally the results shown in this paper must also be an incentive for the production of whole karyotype painting techniques (M-FISH, Sky-FISH) to improve identification of cattle chromosomes.

\section{Authors' contributions}

L.D.L, M.Z. and L.M. performed data analysis. P.M performed mathematical analysis. J.P. performed simulation analysis. P.P. performed data analysis, conceived and designed the study. P.M., J.P. and P.P wrote the paper.

\section{Acknowledgments}

We are very grateful to referees for constructive comments on the earlier version of the paper.

\section{References}

Benet J., Oliver-Bonet M., Cifuentes P., Templado C., Navarro J. (2005). Segregation of chromosomes in sperm of reciprocal translocation carriers: a review. Cytogenet. Genome. Res. 111, 281-90.

Bonnet-Garnier A., Lacaze S., Beckers J.F., Berland H.M., Pinton A., Yerle M., Ducos A. (2008). Meiotic segregation analysis in cows carrying the t(1;29) Robertsonian translocation. Cytogenet. Genome Res., 120, 91-6. 
Cohen O., Cans C., Cuillel M., Gilardi J.L., Roth H., Mermet M.A., Jalbert. P., Demongeot J. (1996). Cartographic study: breakpoints in 1574 families carrying human reciprocal translocations. Hum. Genet., 97, 659-67.

Cribiu E.P., Di Berardino D., Di Meo G.P., Eggen A., Gallagher D.S., Gustavsson I., Hayes H., lannuzzi L., Popescu C.P., Rubes J., Schmutz S., Stranzinger G., Vaiman A., Womack J. (2001). International System for Chromosome Nomenclature of Domestic Bovids (ISCNDB 2000). Cytogenet. Cell. Genet.. 92, 283-99.

De Braekeleer M., Dao TN. (1991). Cytogenetic studies in male infertility: a review. Hum. Reprod., 6, 245-50.

Ducos A., Revay T., Kovacs A., Hidas A., Pinton A., Bonnet-Garnier A., Molteni L., Slota E., Switonski M., Arruga M.V., van Haeringen W.A., Nicolae I., Chaves R., Guedes-Pinto H., Andersson M., lannuzzi L. (2008). Cytogenetic screening of livestock populations in Europe: an overview. Cytogenet. Genome Res., 120, 26-41.

Dyrendahl I., Gustavsson I. (1979). Sexual functions, semen characteristics and fertility of bulls carrying the $1 / 29$ chromosome translocation. Hereditas. 90, 281-9.

Ferguson K.A., Chow V., Ma S. (2008). Silencing of unpaired meiotic chromosomes and altered recombination patterns in an azoospermic carrier of a t(8;13) reciprocal translocation. Hum. Reprod. 23, 988-95.

Fraccaro M., Lindsten J., Ford C.E., Iselius L. (1980). The 11q;22q translocation: a European collaborative analysis of 43 cases. Hum. Genet., 56, 21-51. 
Goddard ME, Hayes BJ, Meuwissen TH. (2010). Genomic selection in livestock populations. Genet Res (Camb). 92, 413-421.

Gonsalves J., Sun F., Schlegel P.N., Turek P.J., Hopps C.V., Greene C., Martin R.H., Pera R.A. (2004). Defective recombination in infertile men. Hum. Mol. Genet. 13, 2875-83.

Lonergan P., Kommisrud E., Andresen O., Refsdal A.O., Farstad W. (1994). Use of semen from a bull heterozygous for the 129 translocation in an IVF program. Theriogenology. 41, 1379-84.

Mäkinen A., Pitkänen T., Andersson M. (1997). Two cases of reciprocal translocations in domestic pigs producing small litters. J. Anim. Breed. Genet. 114, 377-84.

Massip K., Berland H., Bonnet N., Calgaro A., Billoux S., Baquié V., Mary N., Bonnet-Garnier A., Ducos A., Yerle M., Pinton A. (2008). Study of interand intra-individual variation of meiotic segregation patterns in t(3;15)(q27;q13) boars. Theriogenology. 70, 655-61.

Morel F., Douet-Guilbert N., Le Bris M.J., Herry A., Amice V., Amice J., De Braekeleer M. (2004). Meiotic segregation of translocations during male gametogenesis. Int. J. Androl. 27, 200-12.

Ogur G., Van Assche E., Vegetti W., Verheyen G., Tournaye H., Bonduelle M., Van Steirteghem A., Liebaers I. (2006). Chromosomal segregation in spermatozoa of 14 Robertsonian translocation carriers. Mol. Hum. Reprod. 12, 209-15.

Pinton A., Ducos A., Yerle M.(2004). Estimation of the proportion of genetically unbalanced spermatozoa in the semen of boars carrying 
chromosomal rearrangements using FISH on sperm nuclei. Genet. Sel. Evol. 36, 123-37.

Pinton A., Faraut T., Yerle M., Gruand J., Pellestor F., Ducos A. (2005). Comparison of male and female meiotic segregation patterns in translocation heterozygotes: a case study in an animal model (Sus scrofa domestica L.). Hum. Reprod. 20, 2476-82.

Rodríguez A., Sanz E., De Mercado E., Gómez E., Martín M., Carrascosa C., Gómez-Fidalgo E., Villagómez DA., Sánchez-Sánchez R. (2010). Reproductive consequences of a reciprocal chromosomal translocation in two Duroc boars used to provide semen for artificial insemination. Theriogenology. 74, 67-74.

Rodriguez V., Llambí S., Postiglioni A., Guevara K., Rincón G., Fernández G., Mernies B., Arruga M.V. (2002). Localisation of aphidicolin-induced break points in Holstein-Friesian cattle (Bos taurus) using RBG-banding. Genet. Sel. Evol., 34, 649-56.

Schmutz S.M., Moker J.S., Barth A.D., Mapletoft R.J. (1991). Embryonic loss in superovulated cattle caused by the 1;29 Robertsonian translocation. Theriogenology. 35, 705-14.

Schmutz S.M., Moker J.S., Pawlyshyn V., Haugen B., Clark E.G. (1997). Fertility effects of the $14 ; 20$ Robertsonian translocation in cattle. Theriogenology. 47, 815-23.

Van Assche E., Bonduelle M., Tournaye H., Joris H., Verheyen G., Devroey P., Van Steirteghem A., Liebaers I. (1996). Cytogenetics of infertile men. Hum. Reprod. Suppl 4,1-24. 
Warburton D. (1991). De novo balanced chromosome rearrangements and extra marker chromosomes identified at prenatal diagnosis: clinical significance and distribution of breakpoints. Am. J. Hum. Genet. 49, 9951013.

\section{Figures}

\section{Figure 1 - Simulation of abnormal metaphases}

A wild type male metaphase is elaborated to produce an abnormal chromosome longer than BTA1 $(5 \%, 10 \%$ or $15 \%$ longer) or shorter than BTA29 (15\%, 30\% and 40\% shorter). Moreover the full abnormal situation for longer (5 to $40 \%$ ) and shorter (5 to $40 \%$ ) are displayed. a) wild-type metaphase; b),c) and d) artificially modified metaphases with BTA1 5\%, 10\% and $15 \%$ longer than the original chromosome, respectively. e), f) and g) artificially modified metaphases with BTA25 15\%, 30\% and 40\% shorter than the original chromosome, respectively. $\mathbf{h}$ ) details of the artificial modification brought to BTA1 and BTA26. Arrows in a) show the modified chromosomes. 